\title{
The Usage of Usability Techniques in Scrum Projects
}

\author{
Yuan Jia ${ }^{1}$, Marta Kristin Larusdottir ${ }^{2}$, and Åsa Cajander ${ }^{3}$ \\ ${ }^{1}$ Indiana University, Indiana Avenue 719 Indianapolis, United States \\ jiayuan@umail.iu.edu \\ ${ }^{2}$ Reykjavik University, Menntavegur 1, 101 Reykjavik, Iceland \\ marta@ru.is \\ ${ }^{3}$ Uppsala University, Lägerhyddsvägen 2 Uppsala, Sweden \\ asa.cajander@it.uu.se
}

\begin{abstract}
Over the past decades, usability techniques have been introduced into software development practices. At the same time many software development teams have started to use the agile development process - Scrum - to plan and organize their software projects. The focus of this study is to explore how usability techniques are integrated during software development in Scrum projects. The most commonly used usability technique in Scrum projects is workshops, followed by lo-fi prototyping, interviews and meetings with users, all used by more than half of the participants. The technique that is most frequently used is lo-fi prototyping used by more than half of the participants two to four times a month. All these usability techniques are informal, meaning that these techniques can be used quickly without much preparation. Formal usability evaluation with users is a highly ranked technique by the participants but not commonly used by them.
\end{abstract}

Keywords: Usability techniques, User centred design, user involvement, usability, agile software development, Scrum.

\section{Introduction}

Scrum, as one of the agile software development processes, has been gaining popularity in software development over the last few years to plan and organize software development projects [5]. In Scrum the projects are split up in two to four weeks long periods called sprints, each ending up with a potential shippable product that the end users should be able to use right after delivery. In Scrum self organizing and well compounded software development teams are heavily emphasized, typically with six to eight interdisciplinary team members [7]. The main characteristics of the process are simplicity and speed [1] which possibly is one of the reasons for its popularity in industry. One of the benefits of using agile development processes was claimed to be that customers' needs are taken more into account than when developing software using more traditional processes [7]. Traditional Scrum has been criticized for not involving real users in their software process and for not adequately addressing their usability needs [8]. One of the main conclusions in an extensive literature study on the integration of the usability needs into agile processes is that the 
end user needs have not yet been sufficiently included in the agile development processes [9].

At the same time as Scrum became popular in industry, the term usability emerged during the mid 1980s, and was accepted in the 1990s by the software industry. This was partly as a response to the new challenges that web-based software - to be used by a large number of diverse users - put on IT (Information Technology) professionals. However, the body of knowledge of usability is large and includes various perspectives from usability engineering to more context-oriented approaches and these have not yet been accepted fully by industry [3].

For the past decade, usability techniques used in various areas in the industry have been studied by researchers. For example, Venturi \& Troost [10] studied how UserCentered Design (UCD), one of the main approaches in the usability field, was integrated in software development. Larusdottir et al. [5] studied the effect of using a particular development process in industry on the use of user involvement techniques. In that study, about half of the participants were using their own process to plan their work and about one third were using Scrum as their development process. When asked about, if usability is important the participants using the Scrum process were the most negative ones. The most popular user involvement method was meetings with users used by almost all the participants. The use of user involvement methods varied quite extensively according to which process is used for software development. The results from these studies motivated us to examine the use of usability techniques in projects using one particular process, namely the Scrum process.

This paper describes and discusses the results of a survey study on how usability techniques are being used in software projects using the Scrum process to plan and organize the work. The focus of the study is to explore what usability techniques are used, if the usage of one technique is correlated with the usage of the other techniques, how often the techniques are used and how useful they are for IT professionals. The motivation for the study is to gain understanding of what IT professionals need to be able to integrate usability activities more extensively while using the Scrum process in software development.

\section{Background}

Several studies have been conducted on how usability techniques are integrated into software development. It has been explored in some of the studies what software development processes are used, but often it is not analyzed how or if the software process affects how usability techniques were used. In this chapter an overview is given of some of the current literature on how usability techniques have been integrated in software development in industry.

Bygstad, Ghinea, and Brevik [3] surveyed professionals working at Norwegian IT companies to investigate the relationship between software development methodologies and usability activities. In their findings, there was a gap between intention and reality. The IT professionals expressed interests and concerns about the usability of their products, but they were less willing to spend resources on it in industrial projects with time and cost constraints. The results of their survey also 
revealed that the IT professionals perceived usability activities and software development methods to be integrated, which the authors believed is a positive sign.

Bark et al. [2] conducted a survey on the usage and usefulness of HCI methods during different development phases. They examined whether the type of the software projects had any effects on HCI practitioners' perception of the usefulness of the methods. The results show that there was fairly little correlation between the frequency of using a particular technique and how useful it was perceived by the HCI practitioners. One conclusion in the study is that HCI practitioners tend to have a personal and overall evaluation of the different techniques rather than evaluating the actual usefulness of the methods in their daily work when developing particular software.

An international web-based survey by Monahan et al. [6] reported the state of using several field study techniques and how effective they were considered to be by usability practitioners in education and industry. The results show that more than half of the respondents rated observations as an extremely effective method and about $40 \%$ of the respondents rated user testing as extremely effective. The most influential factor for choosing a method for participants working in the software industry was time constrains.

Venturi, Troost and Jokela [11] investigated the adoption of user centred design (UCD) in software industry. The results of the study show that the most frequently used method was user interviews. Additionally, hi-fi and low-fi prototyping methods were frequently used. Overall, the most frequently used evaluation methods are qualitative, allowing rapid feedback to the design activities using expert and heuristic evaluation or "quick and dirty" usability test methods. The results also show that UCD methods are typically used during the early phases of the product life cycle.

A survey study on the usage of 25 usability techniques was conducted in Sweden by Gulliksen et al. [4]. The results show that the usability techniques that received the highest rating by the usability professionals were those that were informal, involved users and were concerned with design issues. Techniques such as expert-based evaluations and benchmarking that do not involve users, received the lowest ratings by the usability professionals. There was a general agreement among the participants that it is important to integrate usability techniques into the software development process they were using. Some participants mentioned difficulties during the integration, especially those that were using RUP (Rational Unified Process) as their development process.

\section{Research Method}

The research method in this study was a questionnaire-based survey. The questionnaire was distributed to IT professionals who were experienced in using Scrum as their software development process and were using usability techniques in their software development.

In the survey we asked IT professionals about their usage of different usability techniques and how useful they rated the techniques. We define usability techniques 
as the various techniques and methods used in software development to enhance the usability of an IT system.

\subsection{Survey Conduction}

The survey was constructed in QuestionPro, which is an online survey tool (http://www.questionpro.com/). The survey included 41 multiple-choice questions and 5 open questions. The questions were developed according to a literature review and interviews with two IT professionals experienced in using Scrum.

The list of 13 usability techniques was a result from a literature review based on Gulliksen et al. [4], Venturi et al. [11] and Larusdottir et al. [5]. However, not all techniques from these studies were chosen since we did not want the list to be too long and risk a low response rate. We chose 5 data gathering techniques: interviews, workshops, questionnaires, meetings with users and field studies, three techniques often used for analysis: usability goals, scenarios and personas, two types of design techniques: lo-fi prototyping and digital prototyping and three techniques for evaluation: formal usability evaluations with users, informal evaluations with users and one expert evaluation technique, the heuristic evaluation. With this selection we wanted to cover a wide range of usability techniques used in software development practice today. The respondents were asked if they had used any other techniques. Two participants responded to that question naming one technique each.

The questions in the questionnaire were grouped into four sections: (1) Information on the companies/organizations and experience of the respondents, (2) the Scrum process in one particular project, (3) the usability techniques that have been used in the particular Scrum project and (4) open questions on usability activities.

Two pilot tests were conducted in order to enhance the quality of the survey. The participants were experts in HCI working in the software industry and using Scrum as their development process. To estimate the approximate time for taking the survey, the first pilot test was timed without any interruption. An interview was conducted after the test. Think-aloud method was used in the second pilot test to detect problems while answering the survey.

\subsection{Survey Distribution}

The survey link was distributed in three different ways. First, the survey link was sent to an email list of software development companies in the Stockholm and Uppsala area. The email list was provided by the Uppsala Tax Office and Lokaldelen, which is a website offering information on companies in Sweden (http://www.lokaldelen.se/). The second way of sending the survey was through directly contacting the target respondents. In order to get more responses, the survey link was also posted on an online discussion group called Scrum Alliance, (http://groups.google.com/group/ scrumalliance) which is an international forum for IT professionals using Scrum.

During a period of 40 days, from $25^{\text {th }}$ April 2011 to $4^{\text {th }}$ June 2011 , totally 49 respondents responded to the survey and 35 of the participants completed all the questions. 


\subsection{Respondents}

Respondents came from 7 countries around the world. The vast majority, $78 \%$ of the respondents came from Sweden, the rest of them came from China (8\%), USA (6\%), France (2\%), Greece (2\%), Lithuania (2\%) and South Africa (2\%).

About $70 \%$ of the respondents had a university degree either a Master or a Bachelor degree. Fourteen out of 49 respondents were certified Scrum Masters, which is a particular role while using the Scrum process. The result shows that about $30 \%$ of the respondents had programming as their main job role and 20\% responded that usability engineering was their main job role. Around $20 \%$ of respondents had a management role in their projects. Others worked for example on code design (10\%), on requirement gathering (10\%) and on UI design (8\%). None of the participants indicated that software testing or evaluation was their main job task.

About one third of the respondents were employed at companies or organizations having up to 50 employees, one third of the respondents at companies/organizations having 50-249 employees and one third of respondents in companies/organizations that had more than 250 employees. Over $40 \%$ of the IT professionals worked on projects for the Internet or the e-commerce area, around $20 \%$ worked in the IT industry in general, about $20 \%$ were working in particular domains like in the health and medical sector, telecommunication sector or in the financial sector. Respondents also reported some other business types that were not listed in the question.

In the second part of the survey the respondents were asked to select one particular project to give information on. Seventy present of respondents had been working on multiple projects simultaneously for the last 3 months. When asked about the type of the project that they had selected, about $40 \%$ responded that the particular project was web related. About $25 \%$ of the professionals were developing software products for sale, around $15 \%$ were developing software systems for clients and about $15 \%$ were developing internal software systems. The remaining respondents mentioned developing other types of systems, including embedded software systems and hardware systems.

\section{$4 \quad$ Results}

This chapter presents the results on what usability techniques are used by IT professionals, how often they use the techniques, how the IT professionals rate the usefulness of the usability techniques and how the usage of one usability technique is correlated with the usage of other usability techniques.

\subsection{Usability Techniques Used in Scrum Projects}

Before asking about the usability techniques, all techniques were listed and explained to make a common understanding of the steps taken while using the techniques and what the names of the techniques actually meant. In Table 1 the usability techniques are listed according to how many respondents had used them. The results in Table 1 show that workshops are the most commonly used usability technique in Scrum 
projects followed by lo-fi prototyping, interviews and meetings with users. Heuristic evaluation was used by only $11 \%$ of the participants and questionnaires were used by about $20 \%$ of the participants. Evaluations with users are not that common, about one fourth of the participants had conducted informal evaluation with users and about one third of the participants had conducted formal usability evaluation with users.

Table 1. The Usage of Usability Techniques

\begin{tabular}{lrrc}
\hline Usability techniques & Used & Total & Percentage \\
\hline Workshops & 30 & 41 & $73 \%$ \\
Lo-fi prototyping & 20 & 36 & $56 \%$ \\
Interviews & 25 & 46 & $54 \%$ \\
Meetings with users & 21 & 40 & $53 \%$ \\
Scenarios & 17 & 36 & $47 \%$ \\
Digital prototyping & 17 & 36 & $47 \%$ \\
Personas & 15 & 35 & $43 \%$ \\
Field studies & 17 & 40 & $46 \%$ \\
Usability goals & 15 & 38 & $40 \%$ \\
Formal usability evaluation with users & 11 & 36 & $31 \%$ \\
Informal usability evaluation with users & 8 & 35 & $23 \%$ \\
Questionnaires & 9 & 42 & $21 \%$ \\
Heuristic evaluation & 4 & 35 & $11 \%$ \\
\hline
\end{tabular}

\subsection{The Frequency of Using Usability Techniques}

The respondents were asked about the frequency of using the usability techniques during one Scrum project. The results from that question are shown in Table 2.

Table 2. The Frequency of Using the Usability Techniques

\begin{tabular}{lrrrrrr}
\hline Usability techniques & $\begin{array}{r}\text { Once a } \\
\text { week or } \\
\text { more }\end{array}$ & $\begin{array}{r}\mathbf{2 - 3} \\
\text { times a } \\
\text { month }\end{array}$ & $\begin{array}{r}\mathbf{7 - 1 2} \\
\text { times } \\
\text { a year }\end{array}$ & $\begin{array}{r}\mathbf{2 - 6} \\
\text { times } \\
\text { a year }\end{array}$ & $\begin{array}{r}\text { Once } \\
\text { a year } \\
\text { or less }\end{array}$ & $\mathbf{N}^{*}$ \\
\hline Interviews & $9 \%$ & $13 \%$ & $22 \%$ & $44 \%$ & $13 \%$ & 25 \\
Questionnaires & $0 \%$ & $0 \%$ & $0 \%$ & $25 \%$ & $75 \%$ & 9 \\
Workshops & $7 \%$ & $7 \%$ & $25 \%$ & $50 \%$ & $11 \%$ & 30 \\
Meetings with users & $15 \%$ & $10 \%$ & $30 \%$ & $35 \%$ & $15 \%$ & 21 \\
Field studies & $0 \%$ & $0 \%$ & $7 \%$ & $53 \%$ & $40 \%$ & 17 \\
Usability goals & $21 \%$ & $7 \%$ & $29 \%$ & $29 \%$ & $14 \%$ & 15 \\
Scenarios & $24 \%$ & $24 \%$ & $18 \%$ & $24 \%$ & $12 \%$ & 17 \\
Personas & $6 \%$ & $19 \%$ & $13 \%$ & $25 \%$ & $38 \%$ & 15 \\
Digital prototyping & $24 \%$ & $12 \%$ & $6 \%$ & $35 \%$ & $24 \%$ & 17 \\
Lo-fi prototyping & $40 \%$ & $20 \%$ & $15 \%$ & $20 \%$ & $5 \%$ & 20 \\
Formal usability evaluation** & $0 \%$ & $0 \%$ & $18 \%$ & $82 \%$ & $0 \%$ & 11 \\
Informal usability evaluation** & $25 \%$ & $25 \%$ & $13 \%$ & $50 \%$ & $13 \%$ & 8 \\
Heuristic evaluation & $0 \%$ & $25 \%$ & $0 \%$ & $50 \%$ & $25 \%$ & 4 \\
\hline
\end{tabular}

* $\mathrm{N}$ represents the number of respondents who had used the technique in their projects.

** With users participating. 
The technique that is most frequently used is lo-fi prototyping, used once a week by about $40 \%$ of the respondents. About half of the participants use scenarios two to four times a month. About $75 \%$ of respondents who had used questionnaires said that they used that technique only once a year or less frequently. About $40 \%$ of the respondents used personas once a year or less than that. The remaining techniques were used two to six times a year.

\subsection{The Ratings of the Usability Techniques}

The ratings of how useful the respondents find the usability techniques are presented in Table 3. The participants were asked to rate the techniques on a five-point scale from very good to very bad.

Table 3. The Rating of the Usability Techniques

\begin{tabular}{lrrrrrr}
\hline Usability techniques & $\begin{array}{r}\text { Very } \\
\text { good }\end{array}$ & $\begin{array}{r}\text { Fairly } \\
\text { good }\end{array}$ & $\begin{array}{r}\text { Neither } \\
\text { good or } \\
\text { bad }\end{array}$ & $\begin{array}{r}\text { Fairly } \\
\text { bad }\end{array}$ & $\begin{array}{r}\text { Very } \\
\text { bad }\end{array}$ & $\mathbf{N}^{*}$ \\
\hline Interviews & $28 \%$ & $60 \%$ & $8 \%$ & $4 \%$ & $0 \%$ & 25 \\
Questionnaires & $0 \%$ & $33 \%$ & $56 \%$ & $11 \%$ & $0 \%$ & 9 \\
Workshops & $38 \%$ & $62 \%$ & $0 \%$ & $0 \%$ & $0 \%$ & 30 \\
Meetings with users & $38 \%$ & $57 \%$ & $5 \%$ & $0 \%$ & $0 \%$ & 21 \\
Field studies & $59 \%$ & $29 \%$ & $12 \%$ & $0 \%$ & $0 \%$ & 17 \\
Usability goals & $53 \%$ & $20 \%$ & $27 \%$ & $0 \%$ & $0 \%$ & 15 \\
Scenarios & $35 \%$ & $59 \%$ & $0 \%$ & $6 \%$ & $0 \%$ & 17 \\
Personas & $40 \%$ & $40 \%$ & $13 \%$ & $7 \%$ & $0 \%$ & 15 \\
Digital prototyping & $59 \%$ & $30 \%$ & $12 \%$ & $0 \%$ & $0 \%$ & 17 \\
Lo-fi prototyping & $50 \%$ & $25 \%$ & $20 \%$ & $5 \%$ & $0 \%$ & 20 \\
Formal usability evaluation** & $73 \%$ & $18 \%$ & $9 \%$ & $0 \%$ & $0 \%$ & 11 \\
Informal usability evaluation** & $25 \%$ & $75 \%$ & $0 \%$ & $0 \%$ & $0 \%$ & 8 \\
Heuristic evaluation & $25 \%$ & $50 \%$ & $0 \%$ & $25 \%$ & $0 \%$ & 4 \\
\hline
\end{tabular}

* $\mathrm{N}$ represents the number of respondents who had used the technique in their projects.

** With users participating.

The result reveals that about $75 \%$ of the respondents thought that formal usability evaluation with users was very good and about $60 \%$ rated field studies and digital prototyping as very good. Around half of the respondents said usability goals, lo-fi prototyping worked very well. No participant rated questionnaires as a very good technique.

If the ratings "Very good" and "Fairly good" are combined, the top five rated usability techniques used by IT practitioners are: 1) workshops 2) informal usability evaluation with users 3) meetings with users 4) scenarios and 5) formal usability evaluation with users. 


\subsection{Correlation of the Usage of Usability Techniques}

Some significant and interesting correlations were found among the usage of usability techniques in Scrum projects. Results in Table 4 shows that there were totally six usability techniques, which had significant correlations with field studies. These techniques are interviews, workshops, meetings with users, personas, lo-fi prototyping and heuristic evaluation. Interviews are significantly correlated with meetings with users. Questionnaires are significantly correlated with digital prototyping, formal usability evaluation with users and informal usability evaluation with users. Workshops are significantly correlated with personas. Usability goals are significantly correlated with scenarios. Digital prototyping is significantly correlated with heuristic evaluation. Lo-fi prototyping is significantly correlated with personas and formal usability evaluation with users.

Table 4. Correlation of the Usage of Usability Techniques

\begin{tabular}{|c|c|c|c|c|c|c|c|c|c|c|c|c|c|}
\hline & $\frac{\underbrace{\infty}_{0}}{\underbrace{0}_{0}}$ & 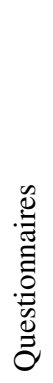 & $\begin{array}{l}\text { aे } \\
\frac{0}{0} \\
\frac{1}{0} \\
3\end{array}$ & 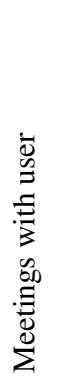 & 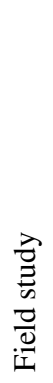 & 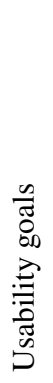 & 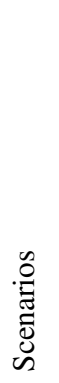 & 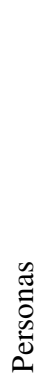 & 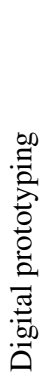 & 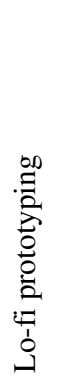 & 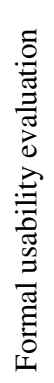 & 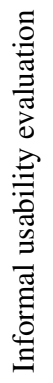 & 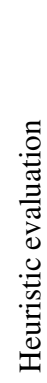 \\
\hline Interviews & & & & + & * & & & & & & & & \\
\hline Questionnaires & & & & & & & & & + & & $*$ & + & \\
\hline Workshops & & & & & + & & & $*$ & & & & & \\
\hline $\begin{array}{l}\text { Meetings with } \\
\text { users }\end{array}$ & + & & & & + & & & & & & & & \\
\hline Field studies & $*$ & & + & + & & & & + & & + & & & * \\
\hline Usability goals & & & & & & & + & & & & & & \\
\hline Scenarios & & & & & & + & & & & & & & \\
\hline Personas & & & $*$ & & + & & & & & + & & & \\
\hline Digital prototyping & & + & & & & & & & & & & & + \\
\hline Lo-fi prototyping & & & & & + & & & + & & & + & & \\
\hline $\begin{array}{l}\text { Formal usability } \\
\text { evaluation }\end{array}$ & & * & & & & & & & & + & & & \\
\hline $\begin{array}{l}\text { Informal usability } \\
\text { evaluation }\end{array}$ & & + & & & & & & & & & & & \\
\hline $\begin{array}{l}\text { Heuristic } \\
\text { evaluation }\end{array}$ & & & & & * & & & & + & & & & \\
\hline
\end{tabular}

+ Represents that the correlation is significant at the 0.05 level.

* Represents that the correlation is significant at the 0.01 level. 
Some of these techniques are related in character like meetings with users and interviews could be conducted in a similar way. Furthermore, interviews are often conducted in the field, so the correlation between field studies and interviews could be expected. On the contrary, the correlation between field studies and heuristic evaluation is rather surprising. The fact that there is a correlation between using the techniques does not mean that these techniques are necessarily used at the same time, but that an IT professional is more likely to conduct heuristic evaluation if that person conducts field studies. Furthermore, a correlation between formal usability evaluation with users and digital prototypes could be expected, but formal evaluations are correlated only with lo-fi prototypes. Questionnaires seem to be used in correlation with evaluation with users, both during formal and informal evaluation. Usability goals seem to be stated in correlation with writing scenarios.

\section{Discussion}

The survey asked the respondents to rate both the usefulness and how often they use the 13 usability techniques. The results show that the top five rated usability techniques used by practitioners are: 1) workshops 2) informal usability evaluation with users 3) meetings with users 4) scenarios and 5) formal usability evaluation with users. This result is partly consistent with the practitioners' perception of the effectiveness of usability techniques in software development projects presented by Gulliksen et al. [4]. The top five rated techniques in that study were: 1) the thinkaloud method 2) lo-fi prototyping 3 ) interviews 4) field studies and 5) scenarios.

Our results show that the top five frequently used usability techniques by IT professionals are: 1) workshops 2) lo-fi prototyping 3) interviews 4) meeting with users and 5) scenarios. This result is also partly consistent with the study from Venturi et al. [11]. Their top five frequently used usability techniques are: 1) user interviews 2) heuristic evaluation 3) qualitative usability evaluations 4) hi-fi prototyping and 5) lo-fi prototyping.

When comparing our results on the top five rated and frequently used usability techniques with those of other studies, it is difficult to draw conclusions regarding the differences. First, the study presented in this paper provided an exact description for each usability technique in the survey, which was not done in the other studies. It may not be viable to compare two ranks of usability techniques when the researchers and the respondents may have had different understanding of what it includes to use a particular usability technique. Secondly, the usability techniques used in the different studies were not the same. For example, workshops are rated as the top usability technique in this study, but workshops are not included in the study by Gulliksen et al. [4]. Moreover, all the usability techniques mentioned in this study were used in software project using the software development process Scrum, but the other two papers are about software development in general.

However, despite these differences some interesting things are worth noticing when comparing the results from the studies. The results from this study show that heuristic evaluation is not often used but the techniques get reasonable ranking in this study and the results from Venturi et al. [11] show that heuristic evaluation was one 
of the top five frequently used techniques. One possible explanation to this difference is that heuristic evaluation may not fit well in the Scrum process. Another possible reason is that the usage of heuristic evaluation as an evaluation method has been decreasing in software development in general, and that the survey results from this study, which was conducted in 2011, indicate a general trend.

The usefulness rated by the participants and the frequency of using the techniques are not correlated. For example, about $75 \%$ of the respondents consider the technique formal usability evaluation with users very good, but only about $30 \%$ used it in their projects. One possible explanation for this inconsistency is that the most important characteristic of Scrum is speed. The duration of one Scrum sprint usually last two to four weeks, but sometimes practitioners need longer time to use a particular usability technique. It usually takes practitioners long time to prepare formal usability testing, recruit participants and conduct the tests, for example.

The techniques digital prototyping and field studies were also rated highly and used two to six times a month. Still, these techniques were only used by one third of the respondents. The technique lo-fi prototyping is more frequently used than the technique digital prototyping. Still the usefulness of digital prototypes was higher ranked than the usefulness of lo-fi prototyping. One of the main advantages of lo-fi prototyping is its quickness and accessibility to any team member in the development process. One explanation could be the focus on speed during Scrum projects.

As shown in the results of the correlation among usability techniques, field studies are significantly correlated with interviews, workshops, meetings with users, personas, lo-fi prototyping and heuristic evaluation. Field studies can help collecting and preparing interview questions and the technique interviews also can provide some valuable information for the process of the field studies. The correlation between field studies and heuristic evaluation is more surprising, because these types of evaluations are rarely conducted in the field. Furthermore, the knowledge that is needed to use the two techniques is not that related. Another technique - formal usability evaluation with users - has strong correlation with the technique questionnaires. One reasonable explanation is that questionnaires can be used to prepare the test tasks or as a post-test survey for the formal usability evaluation with users. Formal evaluation has also a correlation with lo-fi prototypes, which is positive, because it has been suggested in the HCI literature for many years that evaluations should start as soon as possible during the software development.

\section{Conclusions}

This study investigates the integration of usability techniques in software projects using the Scrum process in industry. The findings from the study indicate that the technique workshops are the most commonly used usability technique in Scrum projects, followed by: lo-fi prototyping, interviews, meetings with users and scenarios. Besides, the top five highest rated usability techniques according to usefulness for the IT professionals are: 1) workshops 2) informal usability evaluation with users 3) meetings with users 4) scenarios and 5) formal usability evaluation with users. A novel contribution of this study is that there were significant correlations of 
the usage among different usability techniques in Scrum projects. If IT professionals use workshops they are more likely to use personas is an example of these correlations.

Acknowledgements. We would like to thank all the participants in the study that took their time in answering the questionnaire. Additionally, we would like to thank the PhD student Simon Tschirner and the former PhD students Niklas Hardenborg and Stefan Blomkvist in Human Computer Interaction in Uppsala University for taking part in piloting the questionnaire. Furthermore, we would like to thank the research and teaching assistant Tao Yang at School of Informatics in Indiana University in U.S. for his valuable comments when reviewing the paper.

\section{References}

1. Abrahamsson, P., Warsta, J., Siponen, M.T., Ronkainen, J.: New Directions on Agile Methods: a Comparative Analysis. In: Proceedings of the 25th International Conference on Software Engineering. IEEE Computer Society, Los Alamitos (2003)

2. Bark, I., Følstad, A., Gulliksen, J., McEwan, T., Benyon, D.: Use and usefulness of HCI methods: Results from an exploratory study among nordic HCI practitioners. In: People and Computers XIX - The Bigger Picture, pp. 201-217. Springer, London (2006)

3. Bygstad, B., Ghinea, G., Brevik, E.: Software development methods and usability: Perspectives from a survey in the software industry in Norway. Interacting with Computers 20(3), 375-385 (2008)

4. Gulliksen, J., Boivie, I., Persson, J., Hektor, A., Herulf, L.: Making a Difference: A Survey of The Usability Profession in Sweden. In: Proceedings of NordiCHI 2004, Tampere, Finland, pp. 207-215 (2004)

5. Larusdottir, M.K., Haraldsdottir, O., Mikkelsen, B.: User involvement in Icelandic software industry. In: Proceedings of the I-Used 2009 Workshop at INTERACT 2009, Uppsala, Sweden, pp. 51-52 (2009)

6. Monahan, K., Lahteenmaki, M., Mcdonald, S., Cockton, G.: An Investigation into the use of Field Methods in the Design and Evaluation of Interactive Systems. In: Proceedings of the 22nd British HCI Group Annual Conference, People and Computers, pp. 99-108 (2008)

7. Schwaber, K.: Scrum development process. In: OOPSLA 1995 Workshop on Business Object Design and Implementation (1995)

8. Singh, M.: U-SCRUM: An Agile Methodology for Promoting Usability. In: Proceedings of the AGILE 2008 Conference, Toronto, Canada, pp. 555-560 (2008)

9. Sohaib, O., Khan, K.: Integrating usability engineering and agile software development: A literature review. In: Proceedings of the ICCDA 2010 - International Conference on Computer Design and Applications, Qinhuangdao, Heibei, China (2010)

10. Venturi, G., Troost, J.: Survey on the UCD integration in the industry. In: Proceedings of the NordiCHI 2004 Conference, Tampere, Finland, pp. 449-452 (2004)

11. Venturi, T., Troost, J., Jokela, T.: People, organizations, and processes: An Inquiry into The Adoption of User-Centered Design in Industry. International Journal of Human-Computer Interaction 21(2), 219-238 (2006) 